\title{
A FRAMEWORK FOR THE DESIGN BY OPTIMIZATION OF HYDROFOILS UNDER CAVITATING CONDITIONS
}

\author{
Paolo Olivucci $^{1}$ and Stefano Gaggero $^{2}$ \\ ${ }^{1}$ University of Genoa \\ Via Montallegro, 1, 16145 Genoa, Italy \\ e-mail: 3529870@studenti.unige.it \\ ${ }^{2}$ University of Genoa \\ Via Montallegro, 1, 16145 Genoa, Italy \\ stefano.gaggero@unige.it
}

Keywords: Optimization, Cavitation, Hydrofoils, RANSE, OpenFOAM.

\begin{abstract}
Hydrodynamic shape optimization based on CFD calculations can dramatically improve the design of marine devices (i.e. propellers, rudders and appendages) by simultaneously considering opposite objectives and by modeling phenomena that well-established and still widely adopted design approaches (i.e. lifting line and lifting surface) cannot accurately deal with. Cavitation on propellers, for instance and among the others, is one of the most dangerous phenomena. It causes vibration, erosion and it is a source of radiated noise, consequently resulting incompatible with modern propeller design, continuously aimed for higher efficiency, comfort and environmentally safe operations. An accurate selection, firstly, of the most appropriate blade sections is, consequently, of crucial importance at least to limit the side effects of cavitation. In the present work, therefore, a numerical framework for the design by optimization of marine hydrofoils under cavitating conditions is proposed. By combining a parametric description of the hydrofoil shape, the NSGA-II multi-objective genetic algorithm and appropriate flow solvers, new hydrofoil shapes are derived. Objectives of the design are blade sections with enlarged cavitation buckets to increase the cavitation inception speed and to reduce the cavity volume (under the constraint of unchanged delivered lift) with respect to widely accepted NACA66 profiles. Boundary element methods and RANSE solvers (a proprietary Hess \& Smith implementation and the open-source RANSE solver OpenFOAM) are applied in succession in order to verify the influence of the inviscid/viscous nature of the flow on the final optimal hydrofoil shape and of the additional maximum lift/drag ratio objective required in the case of viscous calculations.
\end{abstract}




\section{INTRODUCTION}

In recent years, the design of marine propellers has consistently evolved. From Betz optimum criteria to lifting line and lifting surface computer codes, the definition of the optimal (under certain constraints and objectives) blade geometry results as the massive application of numerical approaches the more sophisticated the more computational resources became available.

Efforts have been made to optimize the propeller geometry increasing efficiency [1] or optimizing the cavitation behavior [2]. The final consequence of this trend was the completely numerical design by optimization approach proposed in [3] for both conventional and unconventional propellers [4][5]. The so-designed propellers showed superior performance with respect to traditional design methodologies thanks to the application of computationally efficient and accurate potential flow solvers based on panel methods and global convergence optimization algorithms to deal with multi-objective and constrained designs, even in the case of high-demanding controllable pitch propellers [3][6]. Efficiency and cavitation, as a matter of fact, are contradictory demands. In order to fulfill the demand of high efficiency and stable sheet cavitation on the suction side of the blade the loading at tip (the pitch, consequently) cannot be too small: tip vortex cavitation, consequently, may occur and the "bursting" of the vortex in the propeller wake represents a source of broadband excitation to the ship structures. On the other hand, an excessive unloading of the tip may cause leading edge separating vortexes (or pressure side cavitation) which erosive action on the propeller tip can be substantial. The optimization of the main geometrical parameters of the propellers (radial distribution of pitch, camber and chord, for instance) based on accurate potential flow solver calculations allowed for a well-balanced choice of the optimal geometry.

The application of potential panel methods, however, was bottlenecked by the inviscid assumption for the flow. If the influence of viscosity can be partially accounted via simplified formulations when global parameters such pitch or chord were modified, instead, when local geometrical variations (blade leading edge radius or blade sectional thickness distribution, for instance) have to be investigated, it is mandatory to make use of more accurate approaches to avoid unphysical solutions. The designs by optimization already proposed [3][4], for instance, dealt only with global variations of the geometry, avoiding excessive modifications of the base profile shape in the light of limiting the influence (only partially controlled by panel methods and empirical formulations) of the viscosity on local flow phenomena. On the other hand, the reduction of cavitation induced pressure pulses and the prevention of cavitation erosion can be efficiently achieved exactly by using better design of the blade sections instead of the simple geometries of B-Series or MAU series, making optimization also of blade sections worth of investigation.

The use of thin and wide sections as the results of increased blade area ratio and reduced loading per unit area is, probably, the simpler way to improve the cavitation behavior of marine propellers. Thinner sections means larger margins against bubble cavitation on the suction side at a cost of a relatively narrow cavitation bucket that increase the risk of sheet cavitation on both blade sides and dramatically reduces the sheet cavitation inception speed when the propeller operates in a spatial non-uniform inflow. Moreover, larger blade area means higher friction losses and lower propulsive efficiency, opposite to the design objectives. The erosive nature of bubble and pressure side cavitation, in addition, influence the design of the propeller blade. A certain pitch and camber combination is always selected in order to always have an enough margin against pressure side cavitation under the worst unloaded condition. The consequence of this design philosophy is that the design operation point in the average ship wake field can no longer be placed at the angle of attack of shock-free entry of 
the profile. The required average angle of attack is, thus, shifted towards the suction side cavitation with an increased risk of large cavitation volumes and hull excitations.

Hence, the development of robust tools to accurately deal also with a better design of blade sections is indispensable to pursue efficient designs with improved cavitation performance. Instead than designing propellers with higher values of blade area, the application of new blade sections, in fact, may overcome many of the critical points of the usual hydrofoil shapes adopted in the propeller design. By using sections with wider cavitation buckets the design point can be brought back close to the shock free angle of attack of the profile, preventing high suction peaks on the suction side leading edge of the blade and increasing the cavity inception speed. NACA profiles [7], from this point of view, represent a substantial improvement with respect to traditional section series and, despite they were designed for wing sections of airplanes, they are widely accepted in propeller design. The almost flat pressure distribution on the suction side, achieved with the design assumption of the largely adopted a $=0.80$ camber distributions, allows for a relatively wide cavitation bucket, partially limited by the non-optimal response to negative (lower than the ideal) angles of attack. Even better cavitation performance could be achieved by employing different camber and thickness distributions for the evaluation of which, however, advanced design tools are required.

The first attempts to wider the cavitation bucket of hydrofoils for marine propellers date back to the eighties when Shen [8] applied the Eppler's method [9] for the design of improved hydrofoil shapes. Successful application were those by Kuiper [2] and Dang [10], both in the case of hydrofoils and propellers. A non-linear design of the so-called "new blade section" was achieved by Dang [11]. The newly designed hydrofoils were characterized by an unloading of the leading edge to withstand large variations of the angle of attack together with a maximum thickness toward the leading edge as the application of a boundary element method coupled with a thin boundary layer solver. The importance of this design approach was to have pointed out the importance in the design process of the viscous phenomena, in particular the turbulent separation of the boundary layer, which should be accurately taken into account for reliable design and performance improvements. Further advances in wing sections design were those of Amoignon [12], which proposed a gradient-based optimization environment for the definition of optimal wing profiles aimed to the minimization of the drag at constant lift. The solution of the Euler equations with appropriate corrections to account for the laminar flow allowed for reliable designs while by using a RANS equations solver it was possible to consider in the optimization process the turbulent viscosity that may dominate the flow in the particular case of high-lift systems. Even if limited to lift/drag optimization, the application of fully viscous solvers to the design of aeronautical wing profiles showed the obvious advantages that could be expected by using a RANSE solver and that, reasonably, can be expected also in the more demanding case of hydrofoils subjected to the cavitation avoidance constraint.

In the present work, as the first step towards the application of fully viscous approaches to the complete design of marine propellers, a design by optimization of marine hydrofoils is proposed. The design framework consists in a robust parametric description of the hydrofoil shape by using B-Spline curves, a multi-objective optimization based on the NSGA-II algorithm and two different flow solvers, a Boundary element method and the OpenFOAM RANSE tool. Similarly to what proposed by Dang [11], the objectives of the optimization are the widening of the cavitation bucket of a parent hydrofoil (the NACA66 Mod, a $=0.80$ mean camber line, with a maximum camber/chord ratio equal to 0.02 and a maximum thickness/chord ratio equal to 0.04) and the maximization of lift/drag ratio. If, of course for its inherent assumption, the prediction of the frictional drag is pointless when the Boundary element method is applied, with the OpenFOAM RANSE [13] solver it is possible to account 
for the viscosity influence. Two different optimization, therefore, are proposed. The first considers as objective only the widening of the bucket and it is carried out by employing only the Boundary element method. Results are finally checked with the RANSE in order to verify that the optimal solutions under the potential flow hypotheses are still valid when the viscosity is taken into account. The second one is directly based on viscous calculations and, as highlighted above, in addition considers as objective the maximization of the lift/drag ratio at a given delivered lift. Besides, a preliminary validation of the flow solvers is carried out in order to highlight possible limitations and their influence on the predicted cavitation buckets of both the reference and the optimized hydrofoils.

\section{THE DESIGN BY OPTIMIZATION}

The design by optimization consists, essentially, in a reverse design process based on an automatic try-and-error procedure: the hydrofoil shape optimization problem can be formulated as the iterative finding of the set of real valued bounded free variables that control the geometry of the hydrofoil able to minimizes an objective function (cavitation) subject to a set of constraints (lift, for instance). By using any of the available flow solvers, the optimal shape is found by the iterative modification (based on predicted performance) of an initial set of geometries: the design is indirect, rising form the analysis of hundreds (or thousands) of different possible configurations among which identify the better candidates against selected constraints and objectives. This design process, in principle, can be summarized in three steps:

a. A parametric description of the geometry, able to automatically generate suitable variations of the reference design,

b. A computationally efficient CFD method (the Boundary element method or the RANSE solver) for the hydrodynamic analysis of the effects of the shape variations of the thousands of the different candidates under investigation,

c. A reliable and robust derivative-free global convergence optimization algorithm able to find the path to the overall optimum by automatically changing (a) the hydrofoil shape and by computing (b) its performance.

\subsection{Parametric description of the hydrofoil geometry}

The parametric description of the hydrofoil geometry is one of the key point of the optimization process. It is necessary to define automatically each new shape whose hydrodynamic characteristics have to be evaluated in the design process, on the basis of a rather limited number of parameters that turn to be the free variables of the optimization, i.e. the set of values free to be changed in order to identify the optimal design. At the same time, this description has to comply with some "geometrical" constraints: the hydrofoil shape, at least in the case of partially cavitating applications, has to be "faired", avoiding abrupt changes in curvature and slope. A rather well-established way to achieve these results consists in the adoption of B-Spline curves. As proposed in [3][4][5], B-Splines were successfully adopted to model the main geometrical characteristics of conventional and unconventional propellers, providing a robust approach for the definition and the smooth modification, through their control points, of the blade shape. Similarly, also in the hydrofoil case, B-Spline curves were adopted. Rather than directly describing the suction and the pressure side curves, however, it was preferred to model the thickness and camber distributions, following a usually adopted convention in the design of marine propellers. An example of the B-Spline approximation of the parent NACA66Mod section is proposed in Figure 1. The fifteen free parameters considered in the optimization, together with their allowed range of variation expressed as a percentage of the reference NACA66Mod value, are summarized in Table 1. 


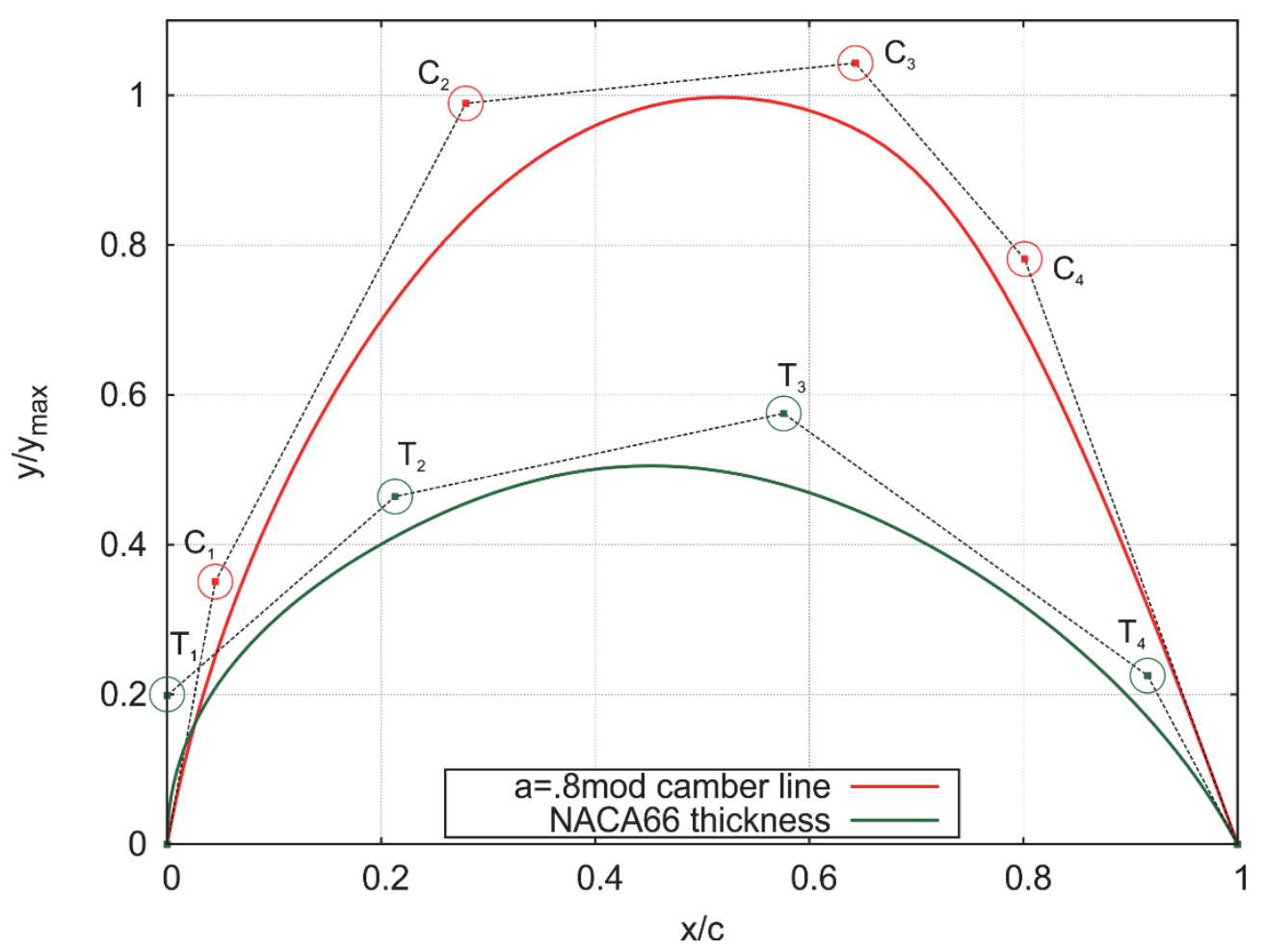

Figure 1: B-Spline description of the reference NACA66Mod non-dimensional half thickness and nondimensional camber distribution and relative control polygons.

\begin{tabular}{lcclcc}
\hline Point & $\Delta \mathrm{x} / \mathrm{c}$ & $\Delta \mathrm{y} / \mathrm{c}$ & Point & $\Delta \mathrm{x} / \mathrm{c}$ & $\Delta \mathrm{y} / \mathrm{c}$ \\
\hline $\mathrm{C}_{1}$ & $\pm 40 \%$ & $\pm 10 \%$ & $\mathrm{~T}_{1}$ & - & $+50 \% /-20 \%$ \\
$\mathrm{C}_{2}$ & $\pm 40 \%$ & $\pm 10 \%$ & $\mathrm{~T}_{2}$ & $+50 \% /-20 \%$ & $+50 \% /-20 \%$ \\
$\mathrm{C}_{3}$ & $\pm 40 \%$ & $\pm 10 \%$ & $\mathrm{~T}_{3}$ & $+50 \% / 20 \%$ & $\pm 30 \%$ \\
$\mathrm{C}_{4}$ & $\pm 40 \%$ & $\pm 10 \%$ & $\mathrm{~T}_{4}$ & $\pm 40 \%$ & $\pm 20 \%$ \\
\hline
\end{tabular}

Table 1: Ranges of variation of the free parameters adopted for the hydrofoil shape definition. The $\mathrm{x} / \mathrm{c}$ position of point $T_{1}$ was fixed equal to 0 (no variations allowed) to avoid cusps at the hydrofoil leading edge.

\subsection{Boundary Element Method and RANSE solvers for hydrofoils hydrodynamic analy- sis}

For the solution of the flow around bi-dimensional hydrofoils, two different solvers were adopted. The first one is a potential Boundary element method for incompressible, inviscid and irrotational flows. The current implementation is based on the low-order Smith and Hess [14] approximations. The Laplace equation for the perturbation velocity potential $\phi$ is solved by a superposition of piecewise constant sources and vortexes, distributed only over the hydrofoil surface discretized by using straight segments, which intensity is defined based on the kinematic and the Kutta boundary conditions:

$$
\left\{\begin{array}{c}
\nabla^{2} \phi=0 \\
\partial \phi / \partial n+\mathbf{V} \cdot \mathbf{n}=0 \\
\Delta p_{\text {trailing edge }}=0
\end{array}\right.
$$


The corresponding pressure distribution over the hydrofoil, from which forces are derived, is calculated with the Bernoulli's theorem. The second solver is based on the solution of the RANSE equations for an incompressible fluid:

$$
\left\{\begin{array}{c}
\nabla \cdot \mathbf{V}=0 \\
\frac{\mathcal{D}}{\mathcal{D} t}(\mathbf{V})=\frac{1}{\rho}\left(-\nabla p+\nabla \cdot \boldsymbol{T}_{R E}+\boldsymbol{S}\right)
\end{array}\right.
$$

where $\boldsymbol{T}_{R E}$ is the tensor of Reynolds stresses (computed according to Boussinesq hypothesis) and $\boldsymbol{S}$ is a tensor representative of additional momentum sources. In the present work, the set of equations (2) is solved, on an unstructured finite-volume mesh, by using OpenFOAM [13] and the SST $k-\omega$ turbulence model to account for the additional stresses due to turbulence.

\subsection{Validation analysis}

A preliminary validation of the both the flow solvers has been carried out in order to assess their accuracy and to highlight the possible limitations that could affect the design of optimal hydrofoils in the optimization process. A suitable test case is represented by the prediction of the pressure distribution around a NACA0012 hydrofoil for which accurate measurements are available in [15]. Boundary element method calculations are based on a surface mesh of 1000 panels arranged in a cosinusoidal spacing at leading and trailing edge that is necessary to have a better description of the local high curvature surfaces. RANSE calculations were carried out on a hybrid unstructured mesh arranged with the open source meshing tools GMSH [16]. An example of the computational mesh, with a close-up view at the hydrofoil leading edge, is proposed in Figure 2. The hybrid mesh (about 35000 cells) consists in an inner, near-wall structured part, made up of prism cells to control the boundary layer phenomena, and an external part filled by triangular elements. An appropriate spacing of the cells was adopted in order to comply with non-dimensional wall distances compatible with the high- or lowReynolds formulation of the turbulence model equations. The results of the analysis are summarized in Figure 3, where the predicted pressure distributions, calculated with different second-order accurate discretization schemes (the OpenFOAM limiteLinearV and linearUpwind) and turbulence modeling, are compared with measurements and with dedicated numerical calculations carried out with the NASA CFL3D code [17] and available in [18].

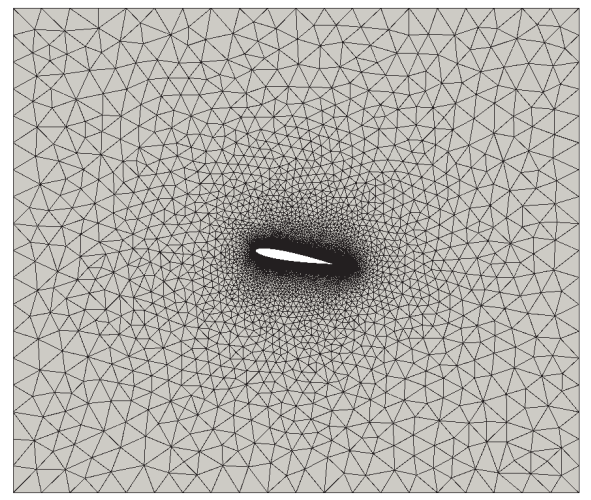

(a) Computational domain

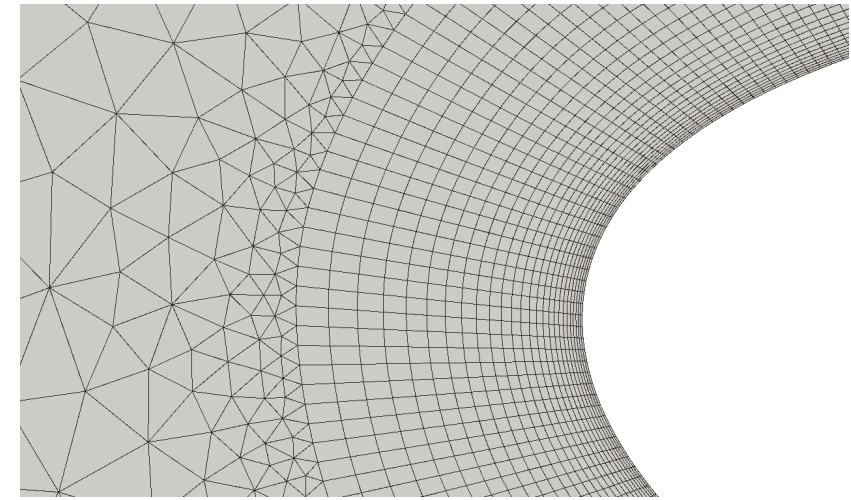

(b) Detail of the mesh at the hydrofoil leading edge

Figure 2: Mesh arrangement for viscous flow calculations - NACA0012 hydrofoil.

The agreement is satisfactory. Both the Boundary element method and the OpenFOAM RANSE solver predict pressure distributions on the suction side close to measurements. At 
the leading edge some differences, however, can be appreciated and have to be considered in the optimization process. Except the NACA CFL3D calculations, that effectively were carried out on a rather different (fully structured, low-Reynolds assumption with $\mathrm{y}+<<1$ ) mesh arrangement, OpenFOAM results, regardless the wall bounded turbulence modeling and the spatial discretization schemes, slightly underestimate the suction pressure peak that, instead, is reasonably foreseen by the CFL3D code. On the contrary, the suction peak predicted by the Boundary element method is higher than both the measurements and the viscous calculations. The cavitation buckets predicted by the Boundary element method are expected, consequently, to be "narrow" (i.e. slightly anticipating, at a given angle, the occurrence of cavitation) than those predicted by viscous calculations.

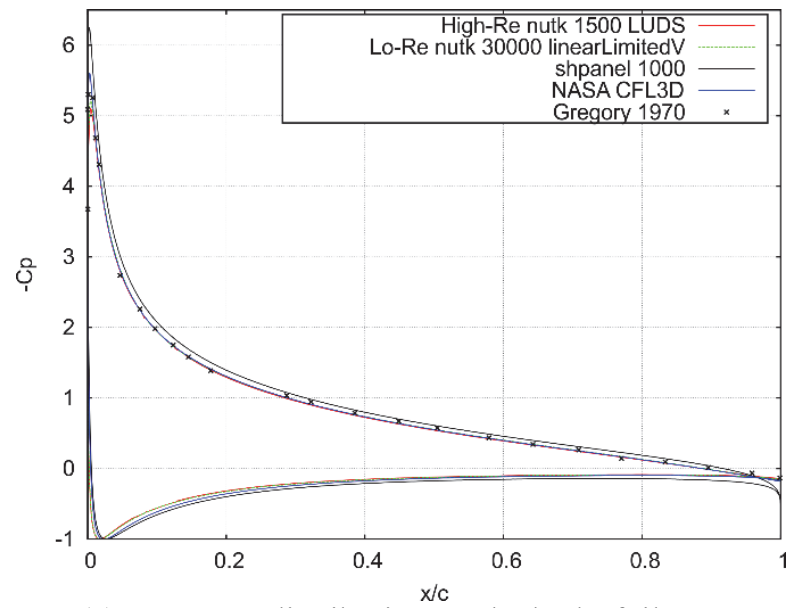

(a) Pressure distribution on the hydrofoil

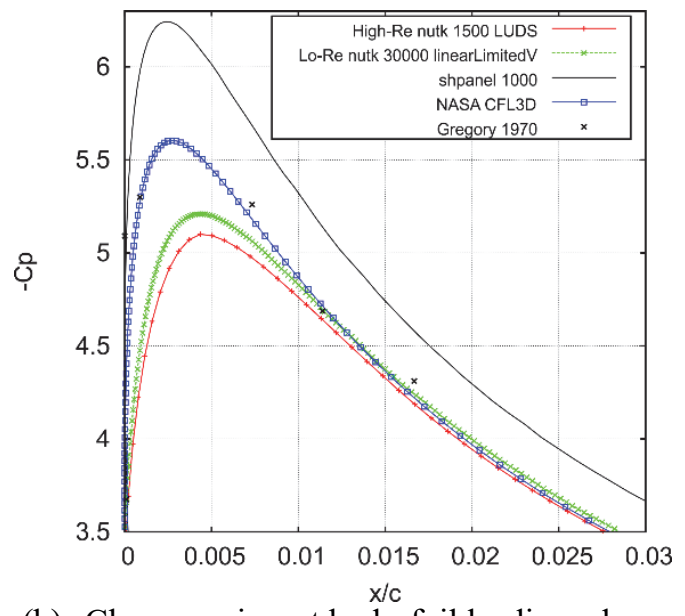

(b) Close-up view at hydrofoil leading edge.

Figure 3: Predicted pressure distribution $\left(-\mathrm{C}_{P}\right)$ on the NACA0012 hydrofoil at $10 \mathrm{deg}$. of angle of attack. Comparison between measurements and numerical calculations.

However, what really matters and drives the convergence of the optimization algorithm is the relative merit and not the absolute value of the predicted performance: only the ability of the numerical approaches to rank correctly different hydrofoil shapes is necessary to perform a design by optimization. The results achieved with this numerical setup (mesh, turbulence assumption and numerical schemes), consequently, may be considered satisfactory, especially in the light of need of computationally fast (few seconds for Boundary element calculations, a couple of minutes for the OpenFOAM RANSE results) approaches to deal with the thousands of cases to be analysed.

\subsection{Optimization process with constraints and objectives}

The optimization process proposed for the design of hydrofoils has a multi-objective nature. Improving the cavitating performance of a hydrofoil presupposes the necessity to simultaneously monitor different and opposite phenomena: suction side sheet cavitation may be reduced by using high values of camber that, in turn, determine an increase of the risk of sheet cavitation on the pressure side with the change of the angle of attack. An increase of the thickness generally turns out to be an effective way to mitigate the suction peaks at leading edge at the cost, however, of an anticipated inception of the bubble cavitation at the hydrofoil mid-chord. In addition, a constraint on the delivered lift has to be forces in order to design hydrofoils which performance are in line with those provided by the reference geometry. A multi-objective optimization algorithm is, consequently, required. In the present calculation, the NSGA-II [19] - Nondominated Sorting Genetic Algorithm was selected. 
Similarly to what proposed by Dang [10], the cavitation bucket represents a useful representation of the cavitating performance for the optimization process. By simply assuming as the cavitation inception the condition in correspondence of which the minimum value of the pressure coefficient on the hydrofoil equals the cavitation number $\left(-C_{P}=\sigma\right)$, any cavitation bucket may be simply computed, regardless the flow solver, by collecting, in correspondence to a sufficient number of different angles of attack, the minimum value of the pressure coefficient. Exactly the same computational idea is under the devised optimization approach. As exemplified in the diagram of Figure 4 , by simultaneously requiring the minimization of the value of the inception points for a set of angles of attack it is possible to drive the selection of any new hydrofoil shape towards the widening of the cavitation bucket, i.e. towards the postponing of the cavitation phenomena.

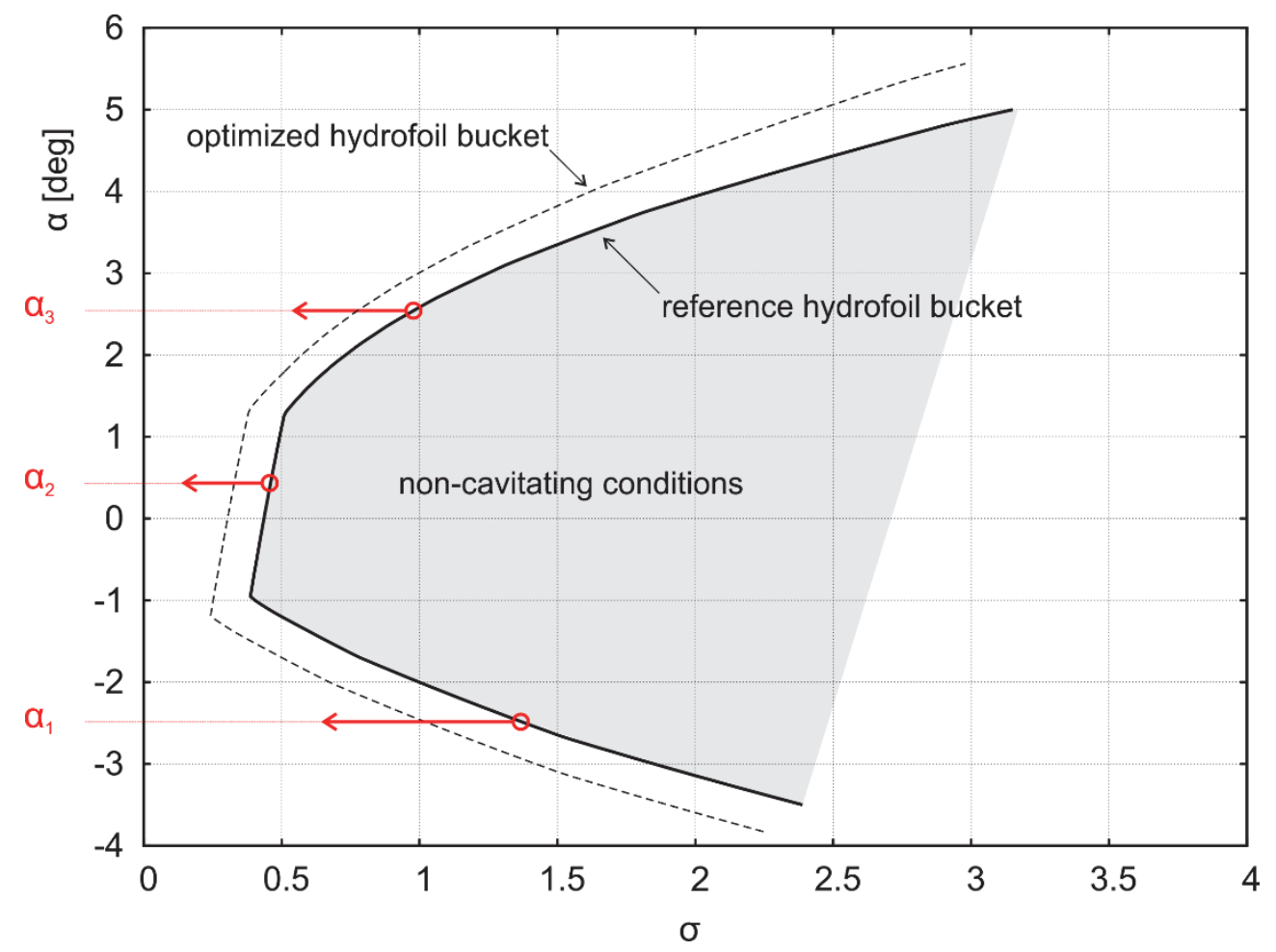

Figure 4: Example of widening the hydrofoil cavitation bucket by simultaneously controlling the cavitation inception at different angles of attack.

The (minimum) number of angles of attack to be monitored in the design process, of course, has to be carefully selected. Having in mind the different type of cavitation a hydrofoil may be subjected to, angles have to be selected in order to let the algorithm monitoring suction and pressure side sheet as well as mid-chord cavitation on the back side. The cavitation bucket of the reference geometry serves as guideline for this selection. The proposed optimization algorithm, in the end, is structured to:

- Minimize the inception value of pressure side sheet cavitation $\left(-C_{P}\right.$ at $\left.\alpha_{1}\right)$,

- Minimize the inception value of mid-chord suction side bubble cavitation $\left(-C_{P}\right.$ at $\left.\alpha_{2}\right)$,

- Minimize the inception value of suction side sheet cavitation $\left(-C_{P}\right.$ at $\left.\alpha_{3}\right)$,

- Deliver a Lift coefficient at a given angle of attack $\alpha_{L i f t}$ equal to that of the reference hydrofoil (with a $\pm 2 \%$ tolerance to speed-up the Pareto convergence), 
- Minimize, only in the case when the OpenFOAM RANSE solver is adopted in the optimization process, the frictional drag.

\section{RESULTS}

\subsection{Design of the optimal hydrofoil - inviscid approach}

The first design was obtained by the application of the Boundary element method as the flow solver in the optimization run. Thus, only a constraint on the lift computed by the Boundary element method itself in the case of the reference NACA66Mod hydrofoil $\left(\mathrm{C}_{\mathrm{L}}=\right.$ $0.26 \pm 2 \%$ at $\alpha=0^{\circ}$ ) was forced. Two different cases were addressed. The first one (case I-inv) was carried out considering as a geometrical constraint the maximum thickness of the hydrofoil which was forced to be equal to the value of the reference NACA66Mod profile. The second run (case II-inv) allows unconstrained variations of the free parameters, resulting in optimized hydrofoils with a maximum thickness and camber different from those of the reference geometry.

The optimization process, as the consequence of the computational efficiency of the Boundary element method, solved 60 thousands different candidate geometries (a population of 100 and 600 generations) for case I-inv. Case II-inv, instead, solved only 10 thousands of different geometries as the combination of a population of 100 members and 100 generations. The performance of case I-inv are summarized in the Pareto diagram of Figure 5. Results are collected in terms of reduction of the inception point of sheet and back cavitation on the $x$ and $y$ - axis while bubbles inception is monitored by the size of the markers. Green points define a subset of geometries having better performance (leading edge sheet cavitation only) with respect to the reference shape that is marked in blue.

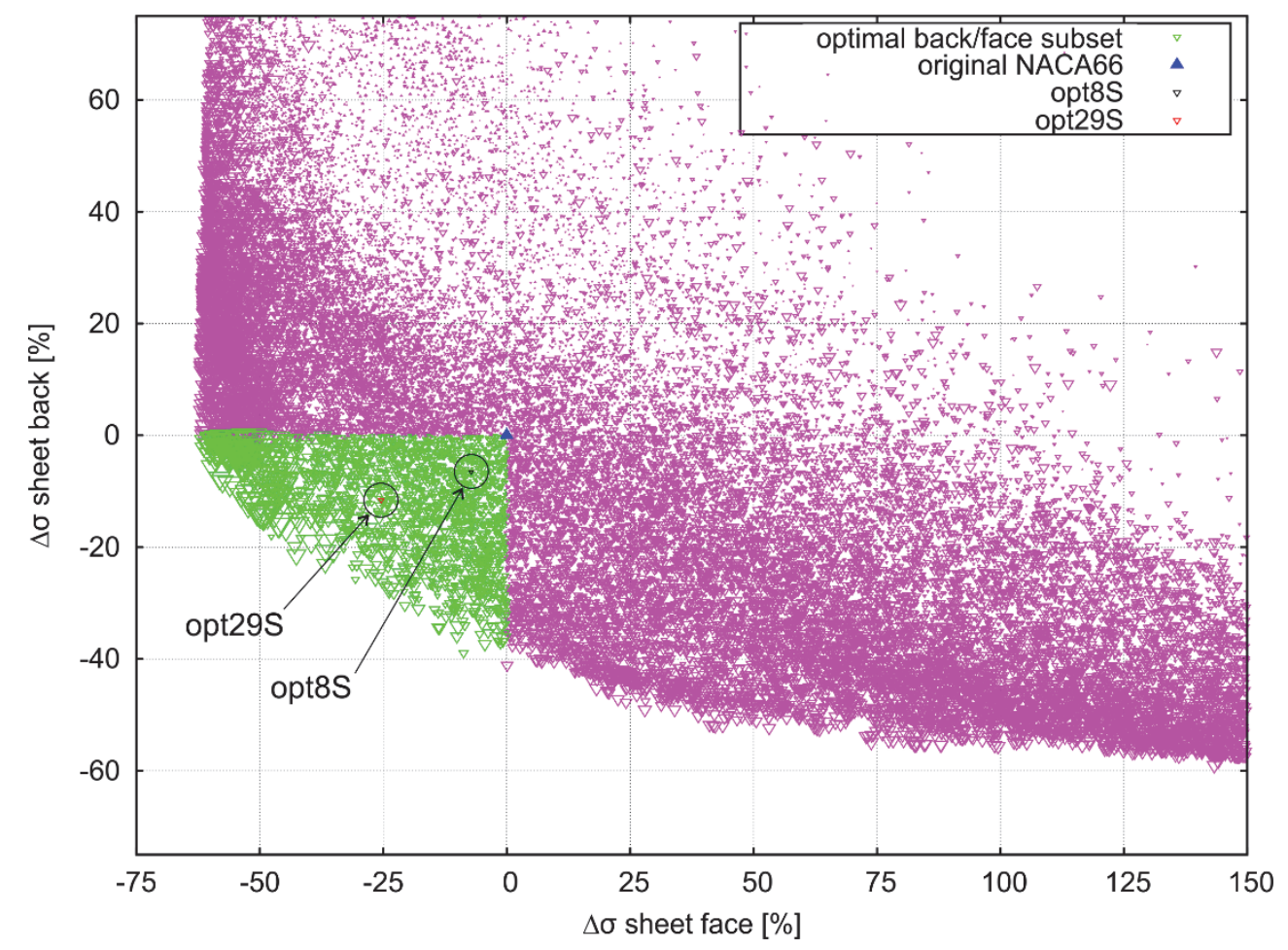

Figure 5: Pareto diagram of the inviscid optimization - case I-inv. 
The improvements (Boundary element method results) achieved through the optimization are remarkable, in particular for what regards the reduction of the suction and pressure side sheet cavitation inception. Bubble cavitation is only slightly enhanced, as better highlighted by the bucket diagrams of Figure 6. Two are optimal hydrofoils extracted from the design by optimization: opt $8 S$ is, among the optimal back/face subset, the geometry with the highest improvement in terms bubble cavitation postponing while opt $29 S$ is a better-balanced geometry that achieve higher improvements for what regards back and face cavitation at the cost of negligible improvements for what regards mid-chord phenomena.
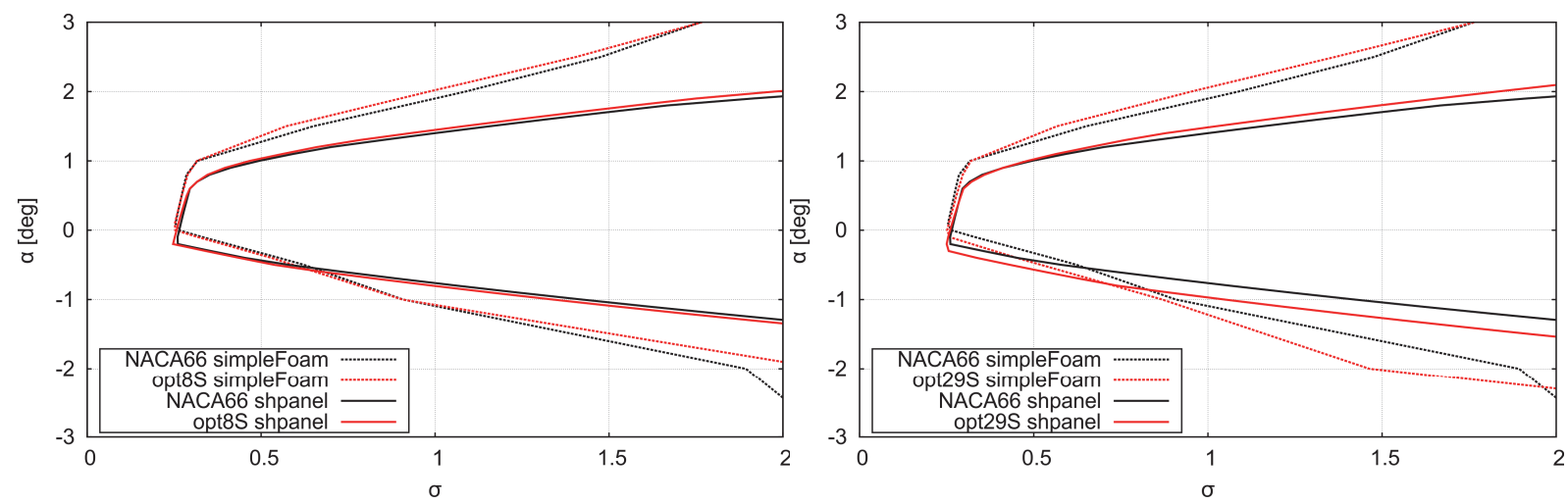

Figure 6: Comparison between the case I-inv optimal (opt8S on the left, opt29S on the right) and the reference (NACA66Mod) cavitation buckets. Boundary element method (shpanel) and RANSE (simpleFoam) calculations.

Essentially the improvements in bubble phenomena, monitored by the panel method, are almost negligible. In the case of the opt $8 S$ geometry the enhancement regards the entire range of angle of attack originally subjected (for the reference NACA66Mod) to bubble cavitation while in the case of the opt $29 S$ only in a narrow range around the $\alpha_{2}$ angle (that adopted in the optimization to monitor bubble cavitation) the optimized hydrofoil provides better performance. The improvements in sheet cavitation inception, instead, are clearly evidenced by the comparison of the bucket diagrams. For the entire range of angle of attack under investigation the optimized geometries provide better performance that result particularly improved in the case of opt29S hydrofoil.
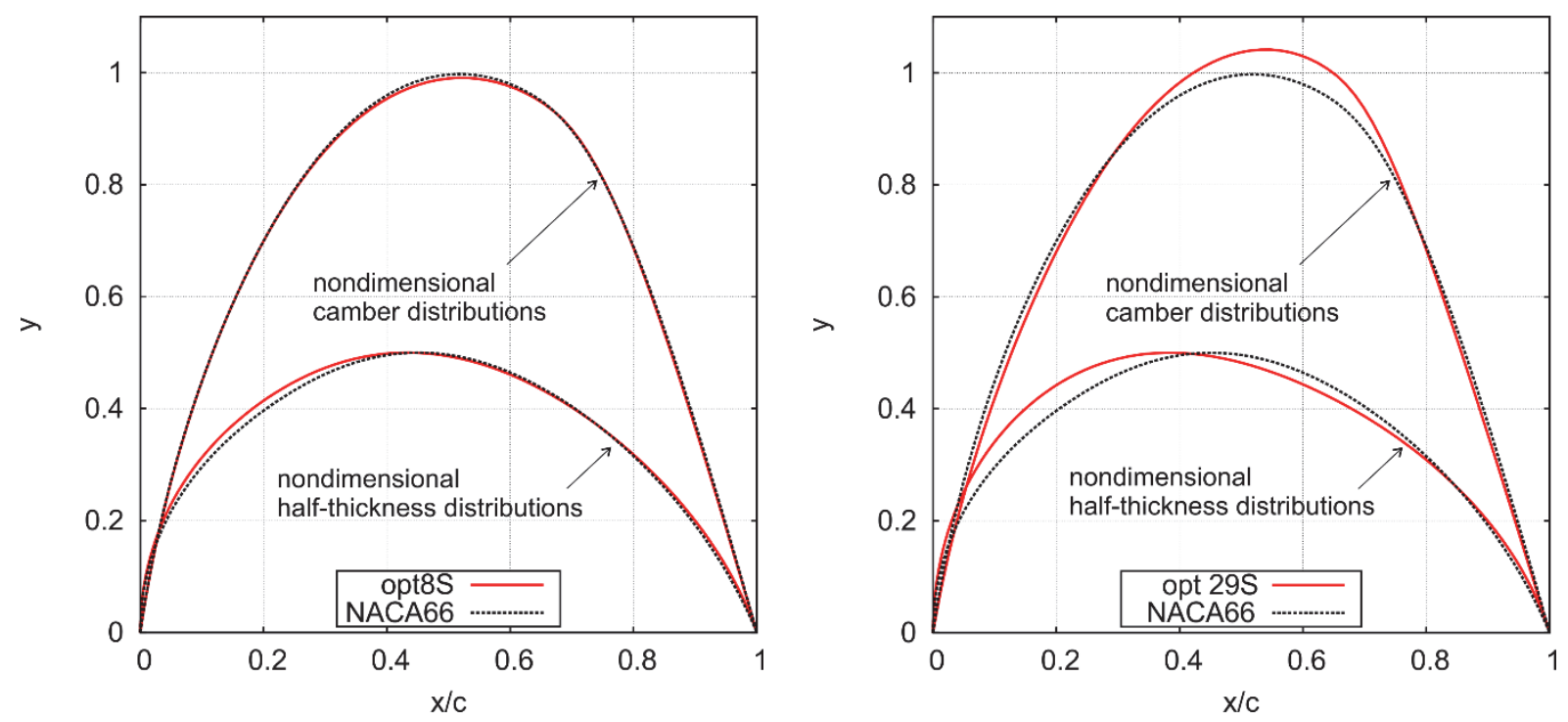

Figure 7: Comparison between the optimal (opt8S and opt29S) and the reference (NACA66Mod) hydrofoil geometries. 
Pressure side inception is significantly postponed, with an appreciable improvement in correspondence of the sharp point that characterize the transition bubble/pressure side cavitation of the reference NACA66Mod shape. These results have been achieved, as expected, by the shift, towards the leading edge, of the maximum thickness, that is particularly evident in the case of the opt29S geometry (Figure 7). This optimized hydrofoil, indeed, effectively produces the higher improvements in terms of leading edge cavitation that are obviously mitigated by locally thicker sections. The improvements evidenced by the optimization are furthermore important from two additional point of view. If the shift of the maximum thickness towards the leading edge is an obvious conclusion for the mitigation of the sheet cavitation, the resulting geometry, as the outcome of the constrained optimization, automatically provides the required performance (lift, for instance) without any worsening, related to the thickness distribution, in terms of bubble cavitation. In addition, the calculations carried out with the OpenFOAM RANSE solver confirm the trends and the ranking of the geometries under investigation evidenced by the panel method. Both the analyzed geometries are overall better than the reference hydrofoil, confirming the outcomes of the design by optimization carried out with the "low" fidelity Boundary element method. In addition, for both the opt8S and the opt $29 S$ hydrofoils, viscous calculations foresee a (very) slightly better performance for what regards bubble cavitation: opt $8 S$, also when analyzed with OpenFOAM, shows a slightly higher increase of the mid-chord cavitation inception speed. Similarly, the cavitation bucket predicted by using viscous calculations in the case of opt $29 S$ is characterized by the substantial improvement in terms of pressure side phenomena already foresee by the inviscid calculations. Even if the absolute values of the cavitation buckets calculated by using the Boundary element method or the RANSE solver are slightly different, the relative merits of one geometry with respect to another are very similar. The Boundary element method, consequently, can be considered a reliable and suitable approach to design hydrofoils under cavitating conditions with the only limitation of the "efficiency" (i.e. the lift/drag ratio) of the newly designed hydrofoils due to the inherent limitations of the potential flow assumption (Table 2).

The second optimization run with the inviscid approach, case II-inv, was carried out by unconstraining the maximum of the thickness distribution. The reference profile under investigation, effectively, is relatively thin: bubble phenomena are naturally postponed (indeed the bubble inception cavitation index of about 0.30 , as from Figure 6 , is very low) at the cost of a leading edge sheet cavitation sensibly worsened. A released maximum thickness should further improve the hydrofoil performance.

The results of case II-inv optimization are summarized in the Pareto diagram of Figure 8 by using the same convention (axis and markers size) adopted for case I-inv. Among the optimal suction and pressure side cavitation subset, two candidates, namely opt 28 and opt 469 , were selected based on bubble cavitation improvements in order to assess the influence of the thickness distribution by analysing, through Boundary element method and RANSE calculations, their complete cavitation buckets.

The complete cavitation buckets of opt 28 and opt 469 (Figure 9) are in line with the results of the optimization: both the hydrofoils provide, for what regards leading edge sheet cavitation, better performance with respect to the reference NACA66Mod. Once again, the relative merits of on geometry with respect to another are identically foresee also by the RANSE calculations, indirectly proving the reliability of the Boundary element method in the optimization process. 


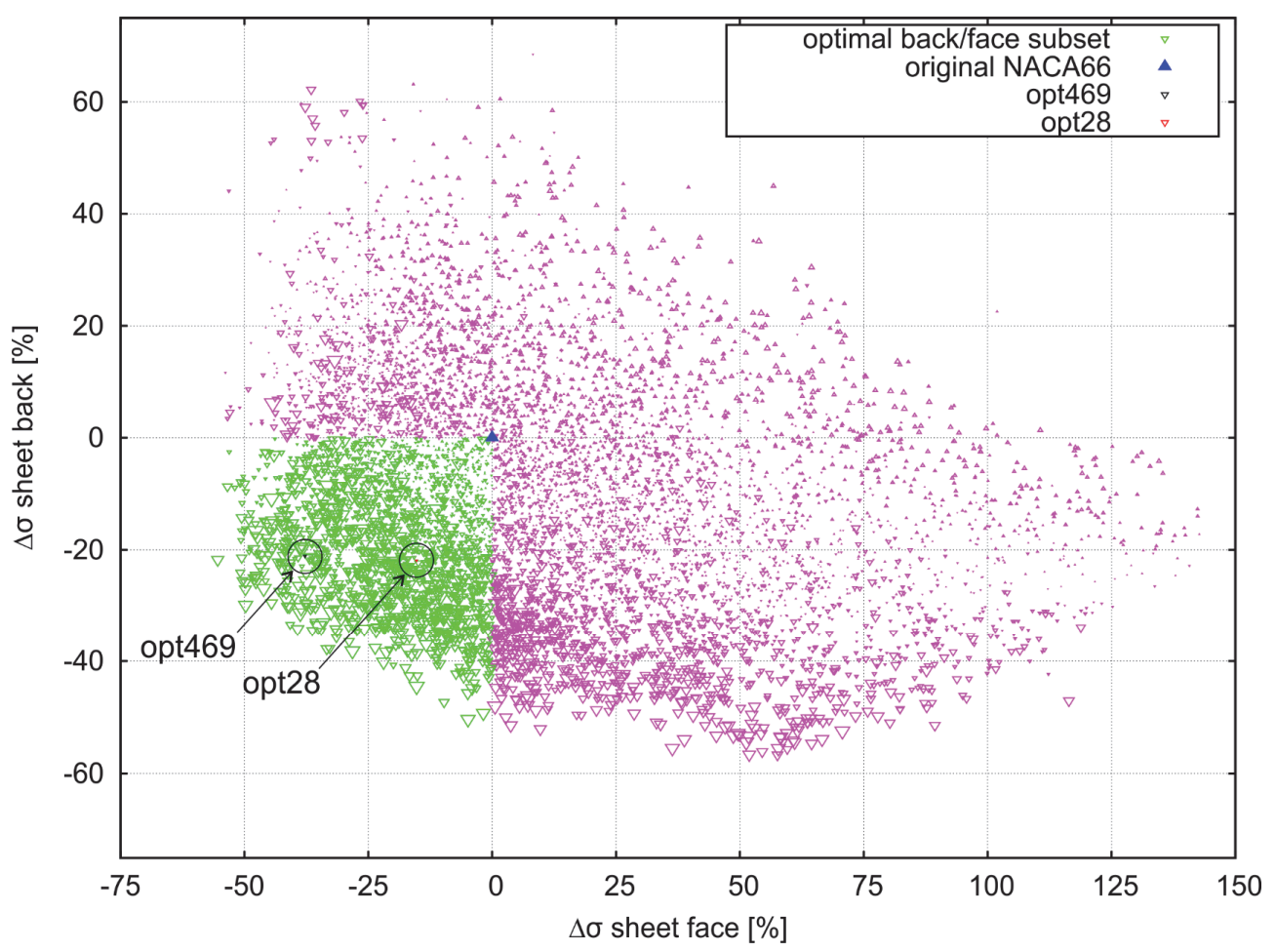

Figure 8: Pareto diagram of the inviscid optimization - case II-inv.

The improvements in terms of sheet cavitation (especially on the face side) of opt469, as it could be expected by comparing the thickness distributions of the hydrofoils shown in Figure 10, are the higher among those of the analyzed geometries (opt8S, opt29S, opt28): opt469 has, indeed, a maximum thickness that is about the $20 \%$ higher than that of the NACA66Mod.
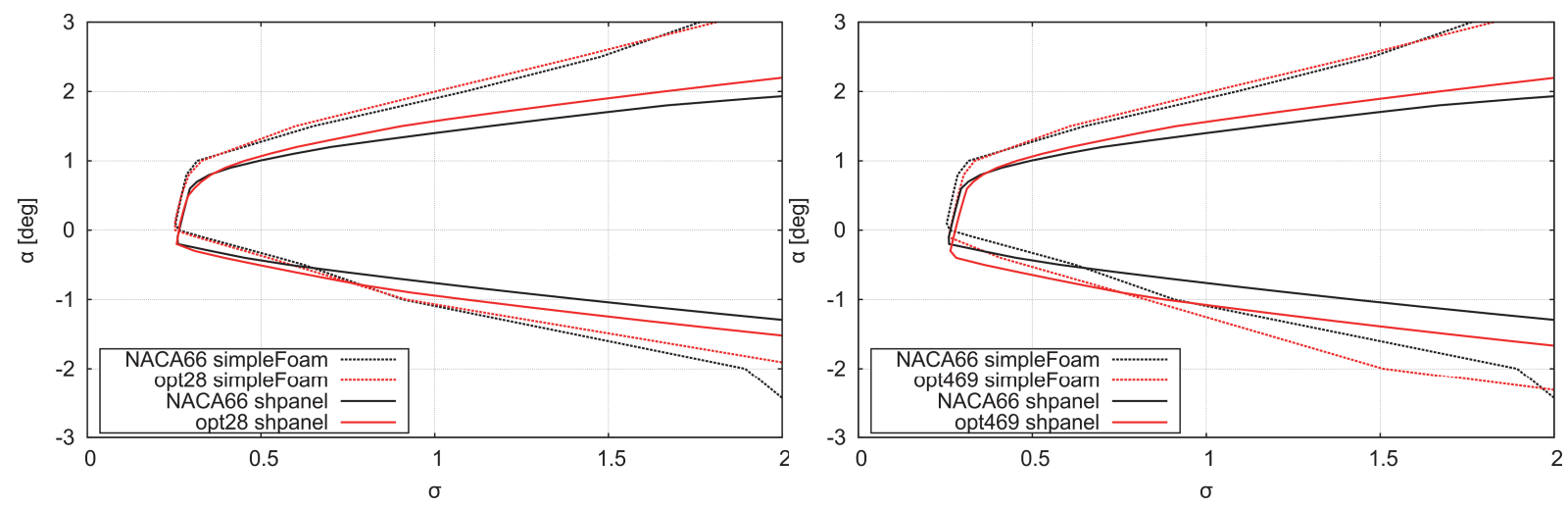

Figure 9: Comparison between the case II-inv optimal (opt28 on the left, opt469 on the right) and the reference (NACA66Mod) cavitation buckets. Boundary element method (shpanel) and RANSE (simpleFoam) calculations.

Moreover, the increased maximum thickness is shifted, as in case I-inv optimization, towards the leading edge. The resulting worsening of the bubble phenomena is exactly evidenced by both the viscous and the inviscid calculations. On the contrary, hydrofoil opt 28 was selected with stricter margin on bubble cavitation. The increase in maximum thickness, that positively acts to widen the cavitation bucket, is limited by the avoidance of bubble cavitation in excess to that of the reference geometry. As pointed out by the cavitation diagrams, the slight increase of thickness that characterize the opt 28 geometry thanks to which its leading edge cavitating performance are improved, is counterbalanced by a slightly lower value of 

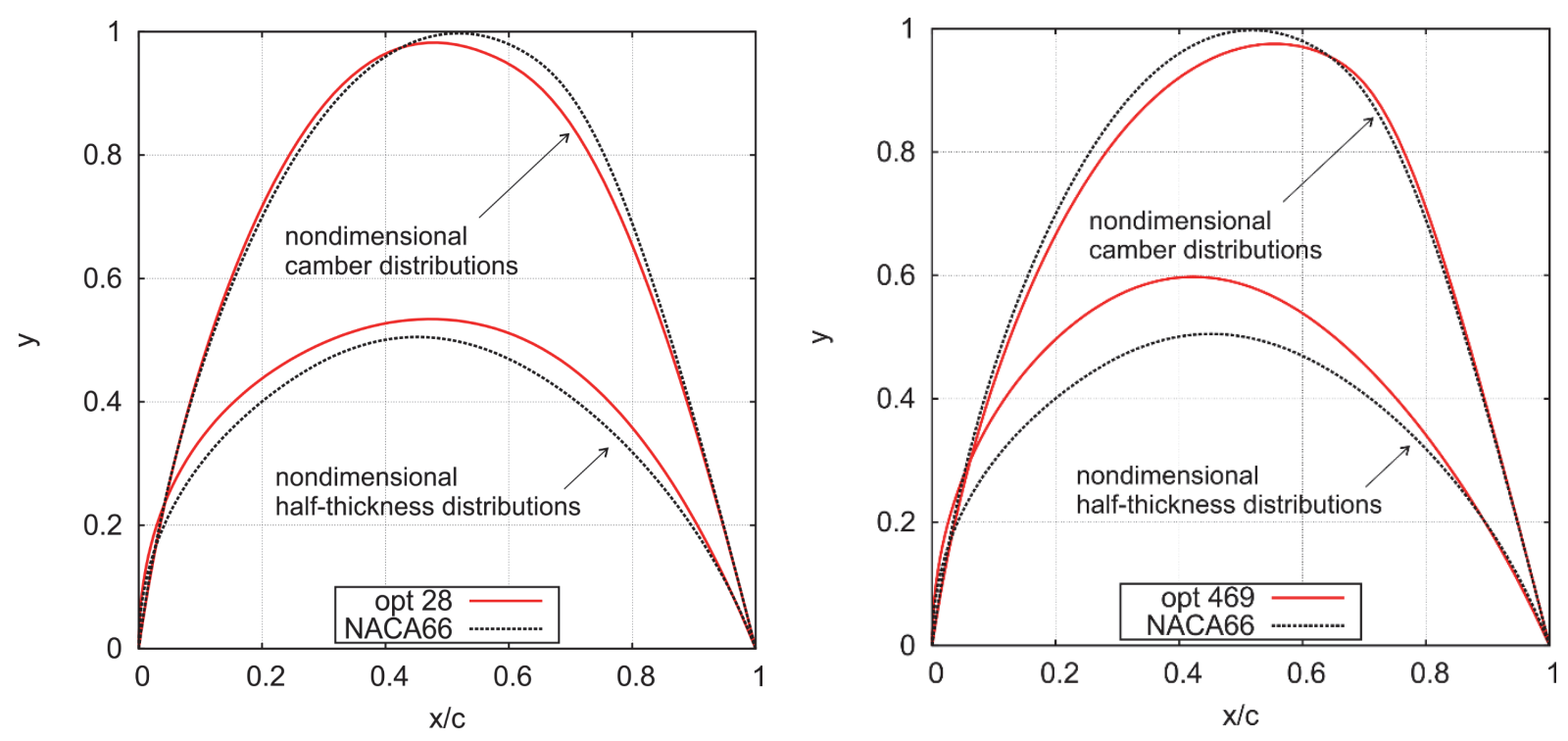

Figure 10: Comparison between the optimal (opt28 and opt469) and the reference (NACA66Mod) hydrofoil geometries.

maximum camber that allows a mid-chord cavitating behavior similar, by both the numerical predictions, to that of the reference profile. As for the case I-inv optimization run, the lift over drag ratios (Table 2), computed a posteriori by using the RANSE solver, for the selected optimal geometries is always lower than that of the reference hydrofoil. The direct application, in the next section, of the viscous solver into the optimization process may definitely guide the design towards even efficient configurations.

\begin{tabular}{lc}
\hline Geometry & Lift $/$ Drag \\
\hline NACA66Mod $-\mathrm{a}=0.80, \mathrm{t}_{\max } / \mathrm{c}=0.04, \mathrm{f}_{\max } / \mathrm{c}=0.02$ & 28.060 \\
Opt8S & 26.932 \\
Opt29S & 26.457 \\
Opt28 & 27.268 \\
Opt469 & 27.910 \\
\hline
\end{tabular}

Table 2: Lift over drag ratios (OpenFOAM RANSE calculations) for the selected hydrofoils at the angle of attack of $0^{\circ}$.

\subsection{Design of the optimal hydrofoil - viscous approach}

An even accurate design by optimization was obtained by the application, directly in the design and not only as a verification tool, of the OpenFOAM viscous RANSE solver. As extensively pointed out in Dang [10][11], viscosity may play an important role. If in the aeronautical field, of course, the main goal of any new designs mainly concerns with the control of the boundary layer and, in turn, of the frictional forces [12], for maritime applications it is important to account for viscosity even if the attention is focused on cavitation. At first, obviously, to have more efficient designs requiring, as an additional objective, the maximization of the lift/drag ratio, for instance. Secondly to have more accurate designs (on the basis, of course, of a high-fidelity flow solver) and account or even exploit any interaction with the boundary layer to widen the cavitation bucket. 
Similarly to what already proposed in the case of the inviscid calculations, two different optimization runs have been proposed. Case I-visc was carried out with a constraint on the maximum hydrofoil thickness (equal to that of the reference NACA66Mod) for a total of 3000 geometries selected by the optimization algorithm while in case II-visc, in analogy with case II-inv, this constraint was neglected and only 1500 geometries were tested. Even if restricted to 2D calculations, the computational efficiency of the viscous solution is orders of magnitude lower than that of the Boundary element methods, forcing the solution only of a fraction of the geometries analyzed by the inviscid approach to comply with a reasonable design time. The amount of collected data is only sufficient to highlight some tendencies and some preliminary optimal results, as evidenced by the Pareto frontiers clearly identified in Figure 11 and 12. In addition to the objectives related to cavitation, monitored on the axes and by the size of the markers, the color scale evidences the lift/drag ratio.

As it can be appreciated by the analysis of the Pareto diagrams and the cavitation buckets of two extracted geometries (opt33SV for case I-visc and opt $8 \mathrm{~V}$ for case II-visc) the inclusion of an additional objective (the lift over drag ratio) and the relatively limited number of evolved generations strongly condition the designs. Both the geometries were selected in the light of a balanced design (simultaneous improvement of the entire set of objectives) trying to limit as much as possible the mid-chord bubble cavitation, that is recognized to be one of the most dangerous cavitating phenomena. As in the case of inviscid calculations, the maximum thickness is shifted towards the leading edge, as shown in Figure 14. An evident difference between $o p t 33 \mathrm{SV}$ and $o p t 8 \mathrm{~V}$ can be, however, appreciated: the latter is characterized by a slightly higher value of maximum thickness, allowed in the optimization process of case IIvisc, and a substantial tapering at the trailing edge.

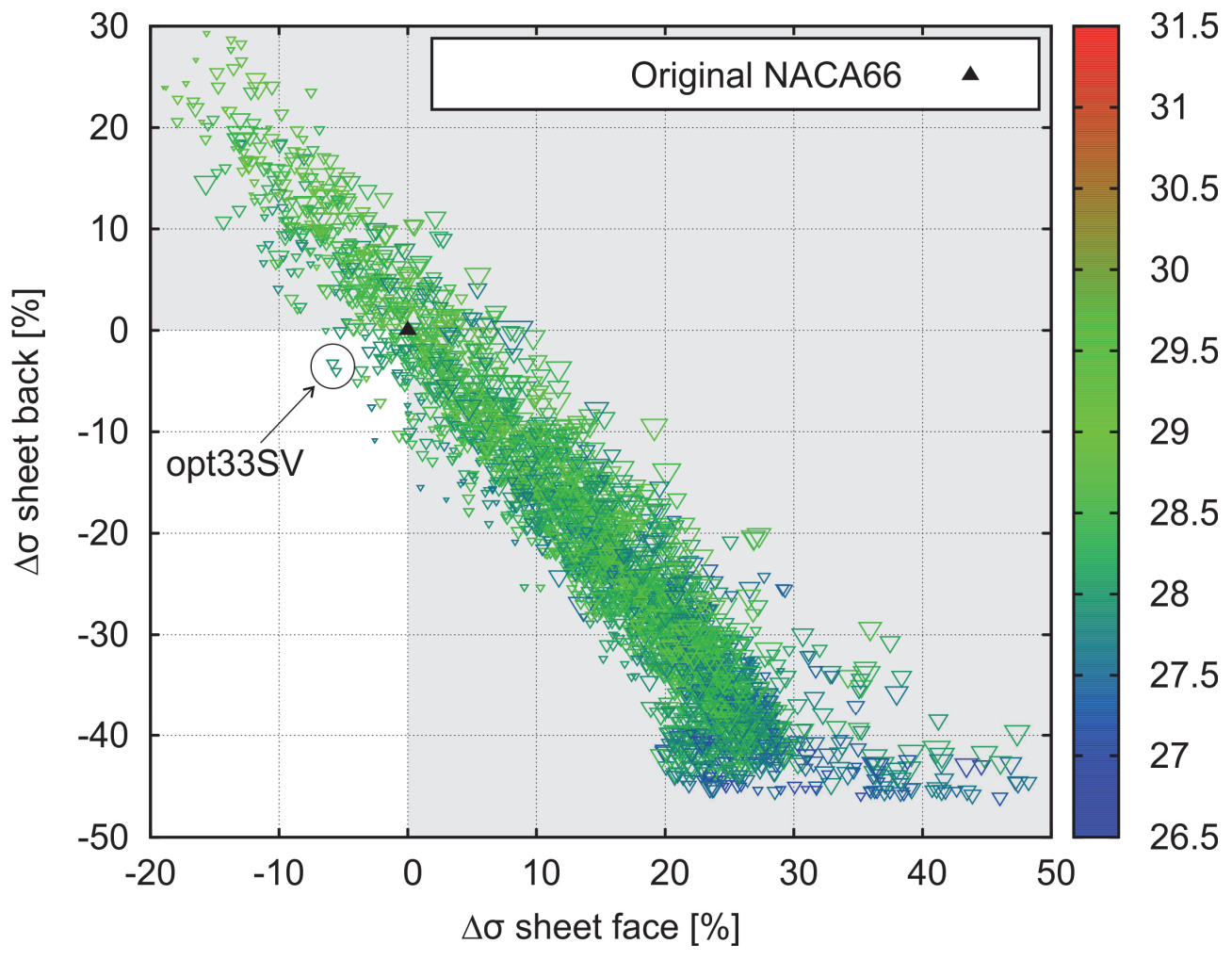

Figure 11: Pareto diagram of the viscous optimization - case I-visc. 


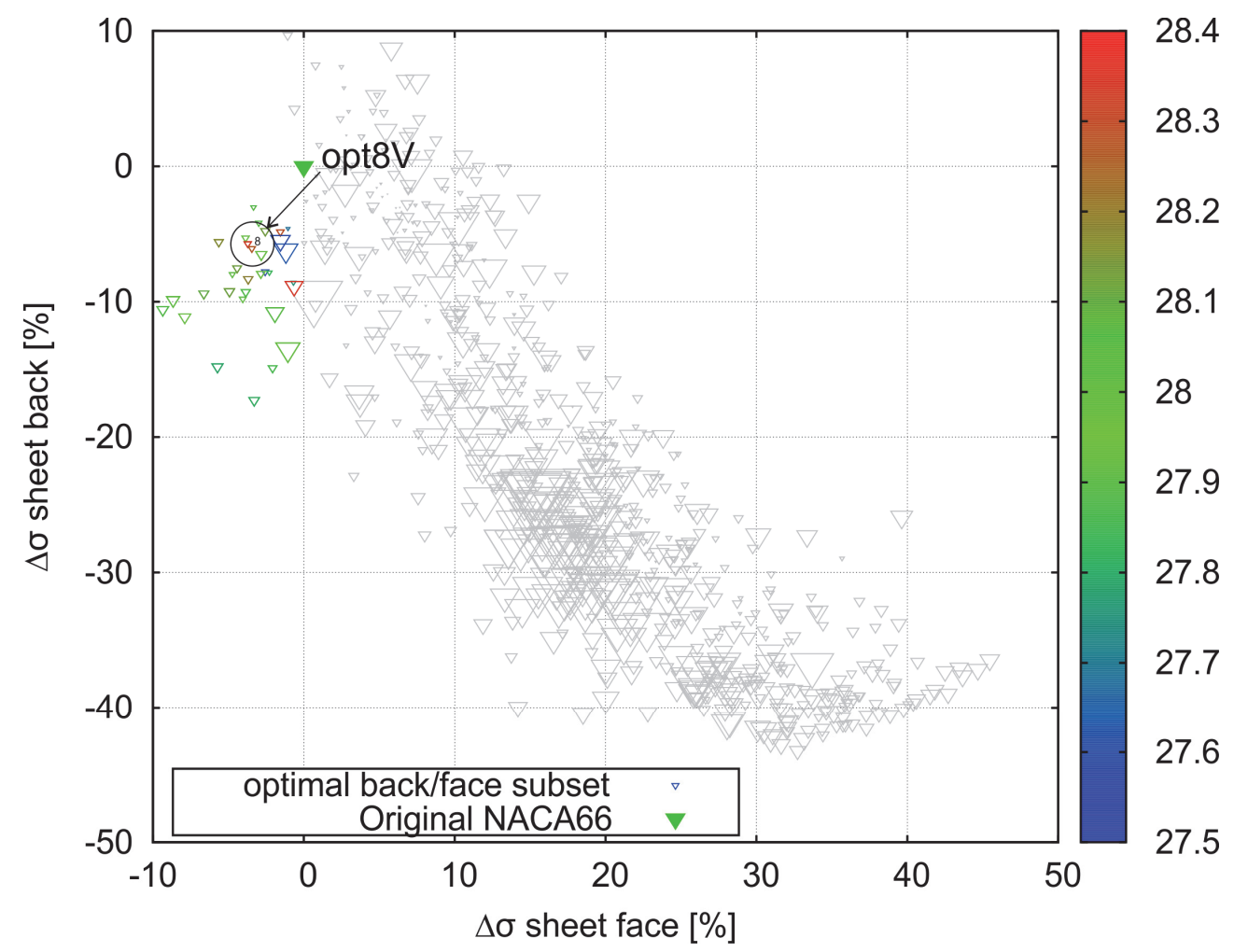

Figure 12: Pareto diagram of the viscous optimization - case II-visc.

The improvements achieved by both the optimizations are highlighted by the bucket diagrams of Figure 13 and by the comparison of the lift over drag ratio of Table 3 . All the cavitating analyses were carried out also with the panel method in order to further verify the reliability of the panel method, eventually only from a comparative point of view, in a case where also viscous were considered for the design.

Optimization of case I-visc (opt33SV), despite the constraint on the maximum thickness, provides slightly better cavitating performance (with respect to both the reference NACA66Mod and the unconstrained thickness optimal shape opt8V) on both the suction and the pressure sides with a negligible worsening, however, of the mid-chord phenomena.
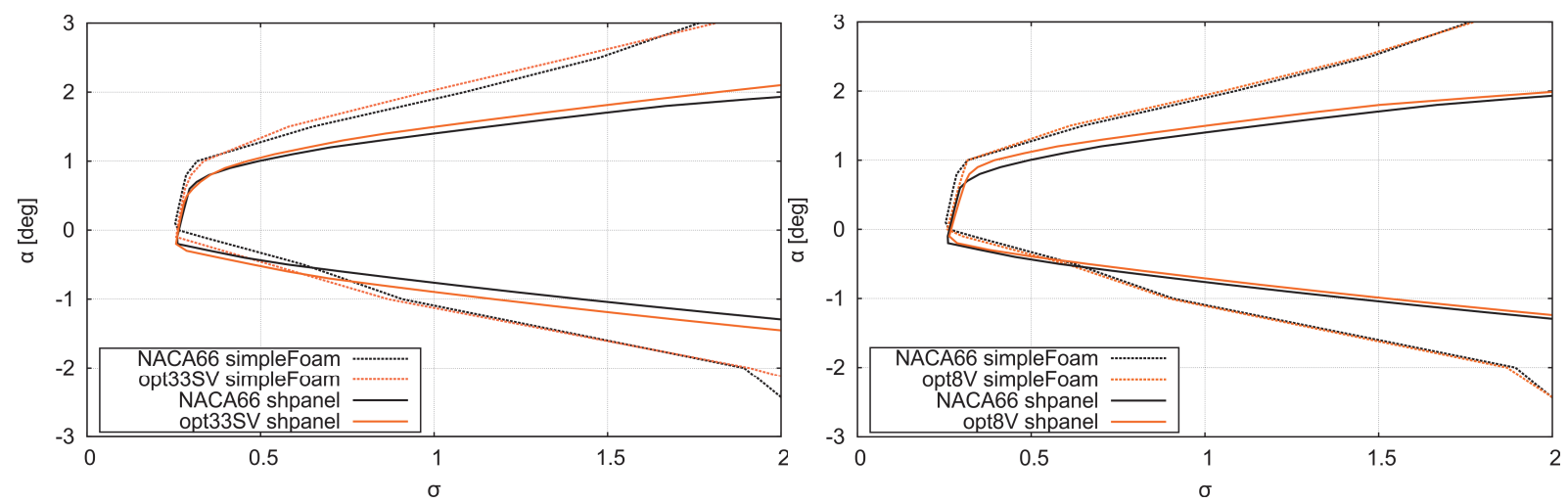

Figure 13: Comparison between the case I-visc optimal (opt33SV on the left), case II-visc optimal (opt8V) and the reference (NACA66Mod) cavitation buckets. Boundary element method (shpanel) and RANSE (simpleFoam) calculations. 


\begin{tabular}{lc}
\hline Geometry & Lift $/$ Drag \\
\hline NACA66Mod $-\mathrm{a}=0.80, \mathrm{t}_{\max } / \mathrm{c}=0.04, \mathrm{f}_{\max } / \mathrm{c}=0.02$ & 28.060 \\
Opt33SV & 28.150 \\
Opt8V & 28.265 \\
\hline
\end{tabular}

Table 3: Lift over drag ratios (OpenFOAM RANSE calculations) for the selected hydrofoils at the angle of attack of $0^{\circ}$.

A single point in correspondence of which the mid-chord phenomena are monitored, as well as only one "positive" and one "negative" angles in correspondence of which the leading edge cavitation phenomena are controlled in the optimization process, may impair the design, producing geometries (i.e. opt8V) only "locally" better. Only the analysis of the entire cavitation bucket may effectively shows the overall fitness of the design that, however, is verified in the case of the opt33SV hydrofoil. With the exception of the higher values of the angle of attack subjected to bubble cavitation, evidenced by the panel method as well, both the suction and the pressure side cavitation performance and the bubble cavitation close to the monitored point are improved with respect to reference geometry. Differently from the inviscid optimizations, that achieved optimal geometries with better cavitating performance but at the cost of a reduced lift/drag ratio (all the analyzed geometries from cases I-inv and II-inv), the opt $33 \mathrm{SV}$ provides, in addition, a slightly reduced value of frictional resistance that results in a slightly improved lift/drag ratio. Opt $8 \mathrm{~V}$, on the contrary, from the cavitation point of view is only negligibly improved. Back and face cavitation is postponed only locally while the overall performance of the hydrofoil are exactly comparable with those of the reference NACA. For values of the angle of attack close to $0^{\circ}$ the pressure side cavitation is even slightly worsened, as well as the mid-chord bubbles that are slightly anticipated with respect to the original geometry. This behavior, foreseen by the Boundary element method too, is balanced by the higher lift over drag ratio that can be achieved with this highly tapered geometry.
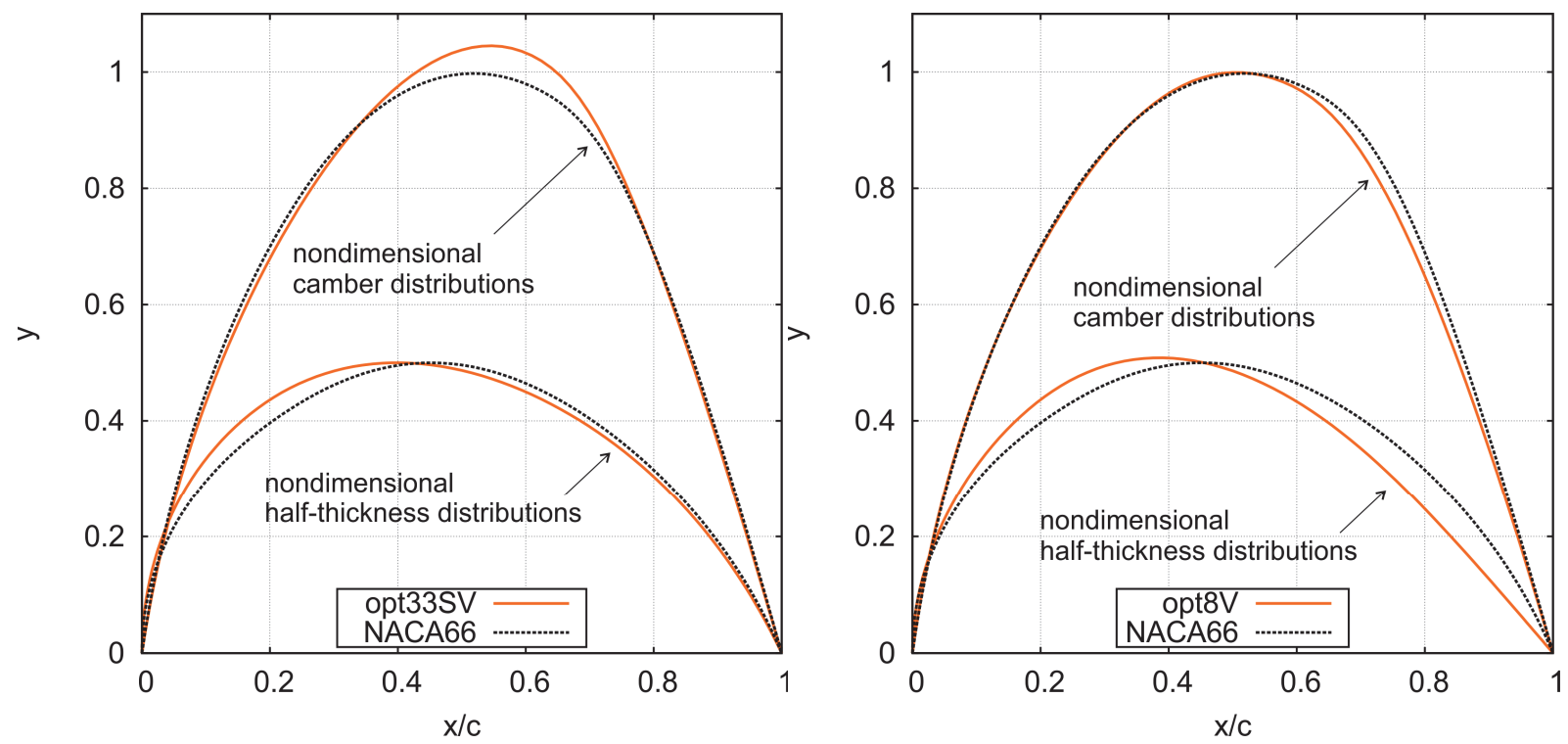

Figure 14: Comparison between the optimal case I-visc (opt33SV on the left), case II-visc optimal (opt8V) and the reference (NACA66Mod) hydrofoil geometries. 


\section{CONCLUSIONS}

A computational framework for the design by optimization of marine hydrofoils under cavitating conditions has been proposed. The design approach relies on a robust parametric description of the hydrofoils geometry, to derive fair and smooth shapes appropriate for subcavitating operations, a multi-objective genetic algorithm and adequate flow solvers (a dedicated Boundary element method and the OpenFOAM RANSE solver) to obtain fast and reliable prediction of the hydrodynamic performance of the bi-dimensional sections under investigation.

Preliminary designs by using the inviscid approach have been proposed, in order to investigate trends and the possible improvements achievable with a very fast computational approach. High fidelity RANSE analyses have been furthermore carried out for the shapes devised by the inviscid calculations in order to verify the robustness of the BEM predictions, also in the light of the validation analysis proposed for a NACA0012 profile. The optimized geometries shown an encouraging widening of the cavitation bucket of the reference NACA66Mod hydrofoil, not only limited to pressure and suction side cavitation. Despite the thinness, that naturally postpones mid-chord bubble phenomena, of the geometries under investigation, a certain improvement of the cavitating performances for angles of attack close to the ideal one has been achieved as well. These results were exactly confirmed by the RANSE calculations, which identically ranked the devised geometries and, consequently, validated the design procedure based on the relative merit of a geometry with respect to the others rather than on absolute values. Only a slightly worsening of the lift of drag ratio, verified a posteriori with the viscous calculations, was observed in the case of the newly designed geometries.

The viscous optimization, further to provide even accurate predictions, was aimed to the definition of optimal hydrofoil shapes also from the "efficiency" point of view, by including as an additional objective of the optimization, the maximization of the lift over drag ratio. The designs by optimization obtained with the viscous flow solver, even if bottlenecked by the relatively small number of geometries analyzed, demonstrated, indeed, the possibility to define geometries that provide better lift/drag ratios. The Pareto diagrams obtained by using the OpenFOAM solver showed rather limited improvements in terms, for instance, of both back and face cavitation with respect to the remarkable improvements evidenced by pure Boundary element calculations. These outcomes are, however, in line with the improvements calculated by the viscous approach when applied to the designs devised by the inviscid solver. The optimization of hydrofoils under cavitating conditions, consequently, results a challenging problem, especially if the parent geometry is an already well-designed hydrofoil. Only small improvements, once calculated with the high fidelity RANSE solver were achieved, but it was possible to obtain an almost overall geometry (opt33SV, for instance) able to postpone suction side and pressure side sheet cavitation and mid-chord bubble phenomena as well (at least for some angles), with an higher lift over drag ratio.

Some points, however, are worth of further analyses and investigations. The relatively small number of geometries tested in the viscous optimization runs, of course, was not adequate to accurately populate the Pareto diagram and clearly identify optimal candidates in a complex, and with contradictory objectives, design. In addition, wider variations of the free parameters should be allowed in order to explore, by using the accurate flow solver represented by the OpenFOAM RANSE tool, even very non-conventional designs, in principle generally avoided when Boundary element methods are applied: out of their range of applications, their inherent assumptions turn into unreliable predictions. Wider free parameters variations, in turn, mean the necessity of a robust finite volume meshing tools able to automatically handle unconventional shapes, especially in present case, for what regards the structured, near- 
wall, part of the mesh. Finally, a more accurate monitoring of the cavitation bucket (by using more than the three angles considered in present calculations) may help in devising geometries with globally (instead than locally) wider bucket diagrams. If the analysis, in the optimization process, of a further set of angles by using the Boundary element method is computationally insignificant, in the case of a viscous driven optimization it additionally reduces the computational efficiency of the design. At the end of the analyses and as the results of all the cross-checked calculations, thanks to comparative nature of the optimization process, the Boundary element method results an efficient tool for a preliminary design of hydrofoils with widened cavitation bucket, restricting viscous calculations only in the case of lift/drag optimizations.

\section{REFERENCES}

[1] S.V. Andersen, P. Andersen, 1986, Hydrodynamic Design of Propellers with Unconventional Geometry, Transaction of the Royal Institution of Naval Architects, 129, 201221, The Royal Institution of Naval Architects, London, 1986.

[2] G. Kuiper, S.D. Jessup, A Propeller Design Method for Unsteady Conditions, Proceedings of the SNAME, SNAME Centennial Meeting, New York, 1993

[3] D. Bertetta, S. Brizzolara, S. Gaggero, M. Viviani, L. Savio, CPP propeller cavitation and noise optimization at different pitches with panel code and validation by cavitation tunnel measurements, Ocean Engineering, 53, 177-195, 2012.

[4] S. Gaggero, J.G. Adalid, M. Perez-Sobrino, Design of contracted and tip loaded propellers by using boundary element methods and optimization algorithms, Applied Ocean Research, 55, 102-129, 2016.

[5] S. Gaggero, C.M. Rizzo, G. Tani, M. Viviani, EFD and CFD design and analysis of a propeller in decelerating duct, International Journal of Rotating Machinery, 2012.

[6] M. Martelli, M. Figari, M. Altosole, S. Vignolo, Controllable pitch propeller actuating mechanism, modelling and simulation, Proceedings of the Institution of Mechanical Engineers Part M: Journal of Engineering for the Maritime Environment, 228, 29-43, 2014.

[7] T. Brockett, Minimum pressure envelopes for Modified NACA-66 Sections with NACA a $=0.8$ camber and Buship Type I and Type II sections, David Taylor Model Basin, Report n. 1780, 1966.

[8] Y.T. Shen, Wing Sections for Hydrofoils - Part 3: Experimental Verification, Journal of Ship Research, 29, 39-50, 1985.

[9] R. Eppler, Direct Calculation of Airfoils from Pressure Distribution, NASA technical translation, NASA TT F-15 417, 1974.

[10] J. Dang, New Blade Section Design and Its Application, Proceedings of the Third International Symposium on Cavitation, Grenoble, France, pp. 301-308, April 1998.

[11] J. Dang, Improving Cavitation Performance with New Blade Sections for Marine Propellers, International Shipbuilding Progress, 51, 353-376, 2004.

[12] O. Amoignon, AESOP - A numerical platform for aerodynamic shape optimization, Journal of Optimization in Engineering, 11, 555-581, 2010. 
[13] The OpenFOAM Foundation. OpenFOAM 2.3.0 users guide, 2014.

[14] J.L. Hess, The Problem of Three-Dimensional Lifting Potential Flow and Its Solution by Means of Surface Singularity Distributions, Computer Methods in Applied Mechanics and Engineering, 4, 283-319, 1974.

[15] N. Gregory, C.L. O'Reilly, Low-Speed Aerodynamic Characteristics of NACA 0012 Aerofoil Sections, including the Effects of Upper-Surface Roughness Simulation Hoar Frost, NASA R\&M 3726, 1970.

[16] C. Geuzaine, J.-F. Remacle, Gmsh: a three-dimensional finite element mesh generator with built-in pre- and post-processing facilities, International Journal for Numerical Methods in Engineering, 79 (11), 1309-1331, 2009.

[17] S.L. Krist, R.T. Biedron, C.L. Rumsey, CFL3D User's Manual (Version 5.0), NASA Langley Research Center, NASA/TM-1998-208444, 1998.

[18] C.L. Rumsey, Turbulence Modeling Resource, NASA Langley Research Center, http://turbmodels.larc.nasa.gov/index.html

[19] K. Deb, A. Pratap, S. Agarwal, T. Meyarivan, A Fast and Elitist Multiobjective Genetic Algorithm: NSGA-II, IEEE Transactions on Evolutionary Computational, 6, 2, 2002. 